\title{
Diagnostic et maintenance prédictive des électrobroches UGV
}

\author{
George Moraru $^{1, a}$, Daniel Brun-Picard ${ }^{1}$, Mustapha Ouladsine $^{2}$ et SÉbastien Mas ${ }^{2}$ \\ 1 LSIS, UMR CNRS 6168, ENSAM, 2 cours des Arts et Métiers, 13617 Aix-en-Provence, France \\ 2 LSIS, UMR CNRS 6168, Domaine Universitaire de Saint-Jérôme, Avenue Escadrille Normandie-Niemen, \\ 13397 Marseille Cedex 20, France
}

Reçu le 14 février 2005, accepté le 13 avril 2005

\begin{abstract}
Résumé - Les électrobroches UGV sont des systèmes complexes et fortement sollicités qui sont la cause de frais d'exploitation élevés : défaillance prématurée initiée par des incidents d'utilisation (chocs, surcharges) ou provoquée par une fuite de fluide de coupe ou de refroidissement; dégradation du cône et des éléments de serrage des porte-outils; dégradation des roulements... Le problème a été étudié conjointement par le LSIS, le LARAMA, COMAU et PCI. Une analyse statistique a été conduite, conjointement à une analyse technologique. La première visait à déterminer les causes principales de défaillance et la deuxième, à identifier les éléments les plus sensibles et les plus critiques. Cet article présente le point de vue statistique ainsi que les orientations de travaux qui en ont résulté, visant à évaluer l'évolution de la dégradation par des mesures in situ, afin de déclencher les interventions de maintenance à bon escient.
\end{abstract}

Mots clés : Usinage / électrobroches / diagnostic / maintenance

Abstract - Diagnosis and predictive maintenance of high speed spindles. Machine tool manufacturers have to permanently increase the guaranteed machine tool availability. This becomes an important requirement on an exceedingly competitive market with a very dynamic evolution. High speed spindles (HSS) are highly complex and highly solicited electromechanical systems, considered as the core element of the machining system. The mechanical or electrical breakdowns of these important elements are to blame for a large amount of manufacturing costs. Online monitoring practices still have difficulties to be integrated because of their price and of their low reliability. Standard monitoring concepts and solutions bring up problems when it comes to high speed cutting because of demanding operating conditions like high speeds and power, high precision, cutting fluid and chips presence, etc. This paper presents a global view on the HSS maintenance problems and an approach proposing a solution in a practical way and by taking into account actual industrial requirements.

Key words: Machining / high speed spindle / predictive maintenance / monitoring

\section{Introduction}

Les machines outils constituent l'élément central autour duquel se structurent les systèmes de fabrication. Leur rôle incontournable dans ces systèmes leur confère un caractère essentiel demandant une fiabilité et une maîtrise du cycle de vie capable de limiter leur coût de fonctionnement global. Un des éléments constitutifs ayant une incidence considérable sur les coûts de maintenance et sur la disponibilité des machines outils est l'électrobroche.

\footnotetext{
a Auteur pour correspondance :

George.Moraru@aix.ensam.fr
}

Les électrobroches UGV ont remplacé depuis bien longtemps les broches classiques et les boîtes de vitesses encombrantes dans la structure des machines outils modernes. Leurs caractéristiques ont répondu à un besoin fort de flexibilité en exploitation et de vitesse accrue des opérations d'usinage. Les électrobroches UGV restent pourtant des éléments sensibles en ce qui concerne la durée de vie car elles sont les sous-systèmes les plus complexes et les plus sollicités d'une machine-outil $[1,2]$.

Le système actuel de maintenance des électrobroches, proposé par les constructeurs de machines outils français a un caractère préventif, basé sur une durée de fonctionnement fixée entre 5000 et 8000 h et sur une mise au 
rebut après 5 révisions. Dans cette configuration le temps d'utilisation est le seul paramètre pris en compte pour estimer l'état de dégradation d'une électrobroche. Ceci entraîne des coûts de maintenance considérables, dus à des interventions sur des broches capables encore de fonctionner ou à des défaillances prématurées initiées par des incidents de fonctionnement qui interviennent avant la révision systématique. On estime que les électrobroches sont responsables d'environ $30 \%$ du coût d'exploitation des machines UGV de l'industrie automobile. La maintenance systématique de l'ensemble du parc de machinesoutils françaises suppose un nombre important d'interventions, pour un coût unitaire de 10 à $15 \mathrm{k} € /$ broche. De plus, toute défaillance intempestive engendre davantage de pertes par un arrêt du cycle de production, paralysé pendant le temps de changement de la broche et de réglage de la machine.

Avec l'objectif de trouver une solution capable de réduire les coûts de maintenance des électrobroches UGV deux fabricants français des machines UGV, PCI et COMAU et deux laboratoires de recherche, le LSIS et le LARAMA, ont démarré conjointement une étude structurée autour de deux analyses :

- une analyse statistique des données existantes sur les défaillances prématurées des électrobroches UGV des deux constructeurs ;

- une analyse technologique de la structure des électrobroches afin d'identifier les éléments sensibles, les mesures possibles et leur pertinence.

Cet article présente l'analyse statistique des pannes répertoriées par les deux constructeurs, les principales conclusions et la stratégie adoptée pour la poursuite de l'objectif déclaré : la recherche d'un ensemble d'indicateurs capables d'améliorer la gestion du cycle de vie des électrobroches et leur disponibilité.

\section{Stratégie du projet et démarche de diagnostic envisagée}

L'objectif de ce projet est de trouver une méthode de suivi de l'état mécanique d'une électrobroche qui puisse améliorer sa disponibilité par une estimation plus précise du risque de panne. Cette méthode doit en même temps respecter des contraintes sévères quant aux moyens effectifs de mise en œuvre, en essayant de limiter à la fois les coûts d'un tel système et les temps d'arrêts de la production.

L'idéal serait de trouver des indicateurs externes et facilement accessibles, ne nécessitant pas de mesures à réaliser sur la machine et permettant d'engager les opérations de maintenance au moment opportun. Une analyse rapide du problème nous a montré qu'il est pourtant difficile de trouver un tel indicateur et que la démarche nécessaire pour le chercher doit passer par des procédures de mesure.

La figure 1 présente la démarche générale de diagnostic avec les logiques associées. La surveillance en ligne

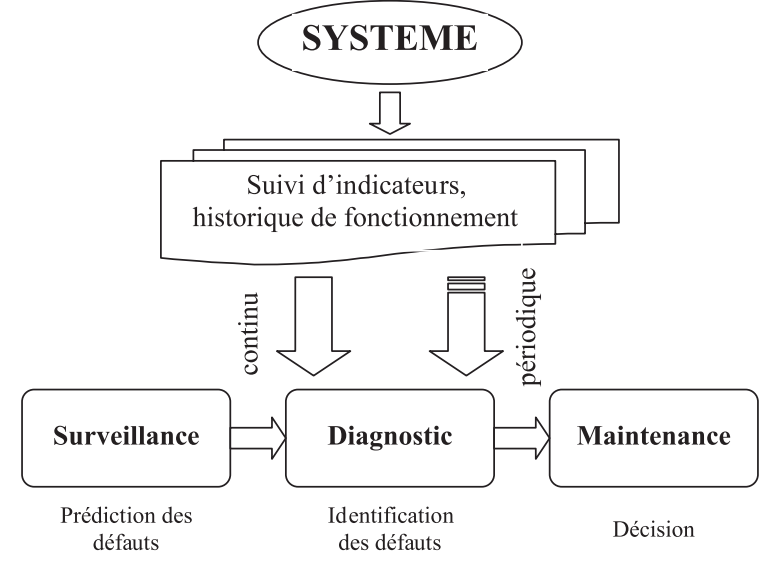

Fig. 1. Démarche de diagnostic et maintenance.

des paramètres de comportement de la broche sera dès le départ écartée des solutions pour des raisons de coûts d'implémentation. De nos jours il existe des systèmes de surveillance vibratoire embarqués [1,3] (enregistreurs type «boîte noire ») mais les industriels ne sont pas convaincus par une telle démarche. On pourrait dire qu'il s'agit d'une décision économique.

Le suivi périodique des électrobroches n'est pas une idée neuve. Il est recommandé et parfois exigé par les constructeurs de broches afin de vérifier les paramètres de fonctionnement de la broche et de détecter les anomalies. Il reste toutefois très pénalisant en raison des périodes d'arrêt demandées. Il s'agit en fait de procédures de mesures simples (mesure de faux rond, force de serrage, vérifications régulières des circuits de refroidissement, lubrification, etc.) mais leur mise en ouvre bloque la machine pour une période de temps considérée par les industriels comme inadmissible.

Un système de mesure et de suivi périodique devrait par conséquent répondre à ces impératifs de rapidité et portabilité. C'est pour cette raison que l'idée adoptée pour résoudre notre problème est de créer un système de mesure portable, capable de caractériser la santé de la broche par le cumul de plusieurs informations :

- caractéristiques de référence mesurées sur la broche neuve; ceci correspond au modèle de bon fonctionnement du système étudié;

- mesures périodiques des paramètres jugés comme importants ; création de leur historique pour les outils de diagnostic type suivi des niveaux globaux;

- enregistrement de l'évolution d'indicateurs pertinents obtenus par une exploitation judicieuse des paramètres pouvant être récupérés sur l'armoire de commande de la machine (puissance moteur, signaux du codeur, nombre de changements d'outils température moteur...).

Afin de réaliser un tel système et de limiter le nombre de paramètres et de mesures à réaliser il faut d'abord déterminer quelles sont les pannes les plus fréquentes et les composants les plus problématiques. Cela nous permettra d'avoir une idée des mesures à réaliser et une image plus exacte du fonctionnement d'une électrobroche. 


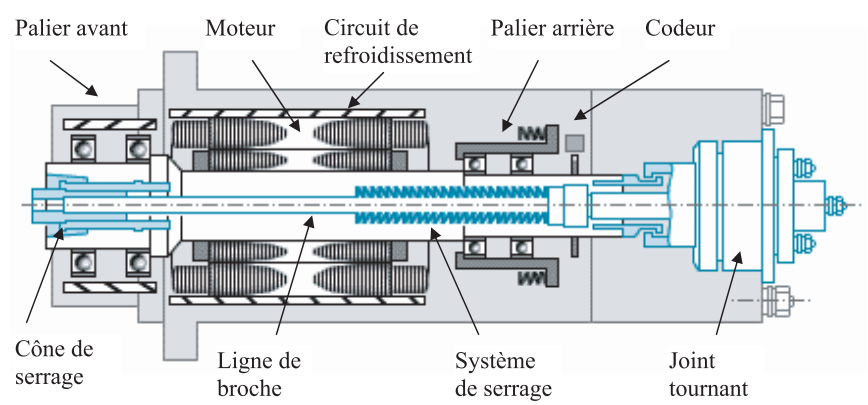

Fig. 2. Structure d'une électrobroche UGV.

\section{Analyse statistique des pannes d'électrobroches}

Toute réflexion sur les moyens à mettre en place pour un système de maintenance prédictive doit passer par une analyse technologique du système étudié et par une analyse préliminaire des données existantes sur les électrobroches installées par les deux constructeurs cités.

COMAU Systèmes France et PCI sont des entreprises spécialisées dans la fabrication des machinesoutils, centres d'usinage et lignes de production automatisée des pièces mécaniques. Leurs principaux clients appartiennent aux industries de l'automobile et de l'aéronautique. La grande majorité des unités de travail sont dotées d'électrobroches UGV de la gamme MFW de la société suisse FISCHER. Les modèles les plus utilisés sont MFM17 (15000 tr.min ${ }^{-1}$ et $17 \mathrm{~kW}$ ) et MFM23 (24000 tr.min ${ }^{-1}$ et $23 \mathrm{~kW}$ ). Ces électrobroches sont dotées d'accouplements HSK A63 et de systèmes de serrage des porte-outils OTT. Les paliers utilisent des roulements hybrides de haute précision.

La figure 2 présente d'une façon très simpliste la structure d'une électrobroche UGV. Pour se faire une idée plus complète de la complexité d'un tel système il faudrait énumérer davantage de sous-systèmes et fonctions assurées : circuits de refroidissement des paliers et du stator, circuits de nettoyage du cône HSK et de lubrification des paliers, capteurs de position du système de serrage, etc. Tous ces sous-systèmes peuvent comporter à leur tour un certain nombre de pièces et/ou ensembles de pièces soumises à des contraintes de fonctionnement pouvant générer des pannes.

Les électrobroches sont des systèmes complexes comportant un nombre important de composants, comparable avec le nombre de pièces de l'ensemble de la structure d'une machine-outil. Elles comportent très souvent des sous-ensembles réalisés par des entreprises spécialisées. Les moteurs sont le plus souvent des moteurs asynchrones à commande vectorielle. Les systèmes de serrage des porte-outils proviennent du fabricant allemand OTT. Un autre élément sensible, le joint tournant, assure la lubrification interne par une alimentation en liquide de coupe en fonctionnement. Il faut donc assurer une étanchéité dynamique à haute vitesse de rotation et pressions d'alimentation, ce qui soulève des problèmes technologiques importants. Les joints tournants sont eux aussi réalisés par des entreprises spécialisées. Les constructeurs des électrobroches se trouvent donc dans une position d'intégrateurs de systèmes sans quitter pour autant celle de spécialiste car des problèmes tout aussi difficiles du point de vue technologique lui sont posés au cours de la conception, de la réalisation et de l'assemblage.

Un produit aussi complexe peut parfois poser des problèmes importants du point de vue de la fiabilité et de la maîtrise globale du cycle de vie. Les machines travaillent dans des conditions difficiles, très différentes de celles d'un atelier d'essais. L'atmosphère, les copeaux, les variations d'efforts de coupe, la présence du liquide de coupe, l'interdépendance et la coordination des équipements de production, le facteur humain représentent tout autant d'aspects à considérer dans l'évaluation correcte d'une solution technologique. Les broches cassent souvent pour des raisons inattendues. Les roulements, en dépit d'une perception généralement fausse mais très répandue, ne sont pas la cause majeure des arrêts des broches. La composition du liquide de refroidissement ou de celui de coupe peut être tout aussi importante, ainsi que le respect des conditions de coupe adéquates.

Un autre aspect important dans l'étude est la notion d'environnement. Il s'agit de prendre en considération des informations concernant le cadre industriel dans lequel les électrobroches travaillent : le niveau de rigueur dans le respect des règles technologiques de chaque client, les opérations d'usinage réalisées (type d'opération, intensité du régime de coupe, matière usinée, nombre de changements d'outils, etc.), respect des règles de maintenance préventive. Il faut aussi être conscient des conditions de travail réelles et des contraintes extrêmement dures imposées par le contexte économique concurrentiel. Les clients n'ont pas toujours le temps d'appliquer ces règles, et les calculs économiques rapides leur montrent souvent que le non respect de ces règles de maintenance peut être moins coûteux que les arrêts demandés.

Les sociétés COMAU et PCI ont déjà mis en place, dès 2001, une procédure de suivi des électrobroches montées sur leurs machines. Ces renseignements, la plupart sous forme de tableaux, contiennent des informations et constituent chacune une base de données sur les constats des clients et/ou sur les expertises effectuées par le constructeur avant la réparation (FISCHER). Nous avons entrepris l'analyse séparée de chaque base de données et une analyse globale afin d'identifier les aspects concordants et les différences.

Après l'étude des bases de données, un nombre de 93 broches répertoriées par COMAU et un nombre de 182 de PCI ont été retenues. Les fiches qui ne semblaient pas offrir suffisamment de données ont été écartées. Les figures 3 et 4 présentent les résultats de l'analyse statistique $[3,4]$.

Le premier constat de l'analyse et le plus évident est que les deux points de vue, client et constructeur, ne sont pas compatibles et cela pour la majorité des électrobroches répertoriées. Cet état des faits peut être expliqué avec une analyse plus fine des notions et 
CONSTATS CLIENTS (COMAU)

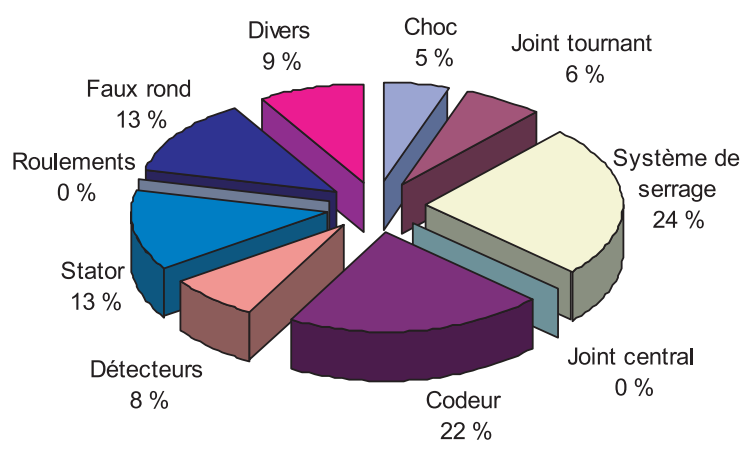

CONSTATS EXPERTISE (COMAU)

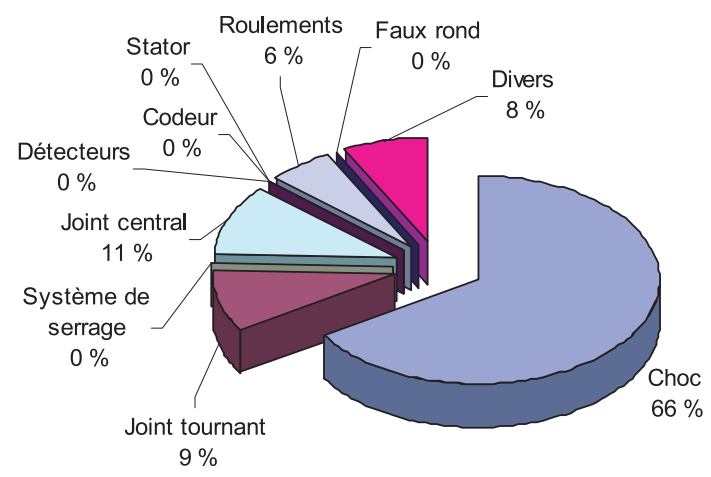

Fig. 3. Analyse des pannes électrobroches (RAC - 93 broches).

CONSTATS CLIENTS (PCI)

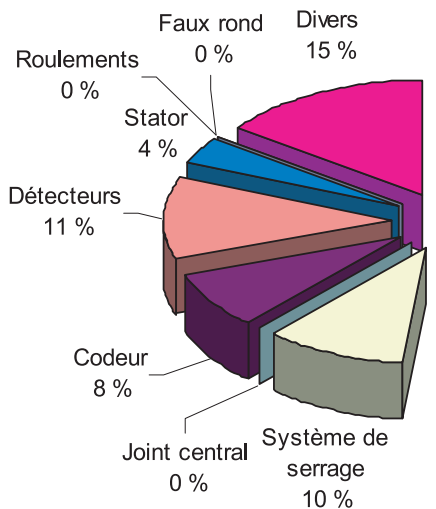

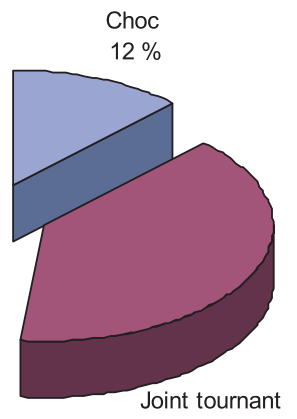

$40 \%$
CONSTATS EXPERTISE (PCI)

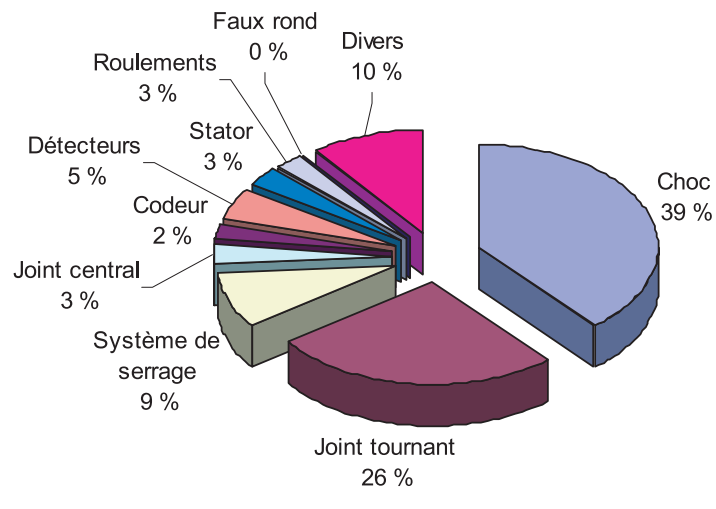

Fig. 4. Analyse des pannes électrobroches (PCI - 182 broches) [5].

termes utilisés par les deux acteurs : le client et l'expert constructeur.

Premièrement, les deux analyses, par les définitions même des pannes, mélangent deux, voire trois notions différentes. Les pannes sont traitées parfois de causes, les symptômes - surtout dans le cas des clients - sont vus comme des pannes ou même des causes primaires. La plupart des constats des clients COMAU portent sur les pannes, en essayant de déterminer l'élément qui a généré l'arrêt de la broche. Cet élément ou sous-système n'est pourtant généralement pas celui qui a initié la panne. Les clients désignent comme responsable de l'arrêt le codeur, parce que le variateur indique une panne codeur par son diagnostic interne. Une analyse plus détaillée, telle qu'elle est pratiquée par l'expert, peut montrer que le codeur a été en fait inondé par une fuite du joint tournant, ou que le faux rond de la roue codeuse est en dehors de la plage acceptable. De la même manière les problèmes du système de serrage sont très souvent liés à des collisions générées par un mauvais alignement du système de changement d'outil. C'est d'ailleurs l'explication de la proportion importante des pannes générées par les collisions ou les chocs provoqués pendant le fonctionnement de la machine. Ceci vient compliquer davantage l'analyse et le fait que les chocs ne provoquent pas toujours une panne immédiate. Un arbre voilé par un choc ou une série de collisions avec le magasin d'outils peut provoquer dans le temps un mauvais fonctionnement du codeur à grande vitesse, le balourd se manifestant dans ce cas par un faux rond dynamique important.

Une seconde observation importante dans notre analyse est que les deux constructeurs n'ont apparemment pas les mêmes problèmes de broches. En effet, les broches de PCI présentent une proportion largement plus importante des pannes générées par le joint tournant que les broches de COMAU. Ceci peut s'expliquer par les différences constructives entre les broches utilisées et entre les joints tournants intégrés. Depuis que l'analyse a été réalisée les problèmes du joint tournant de PCI ont été en grande partie résolus.

Les autres différences s'expliquent par la notion d'environnement. Les broches ne travaillent pas dans le même 


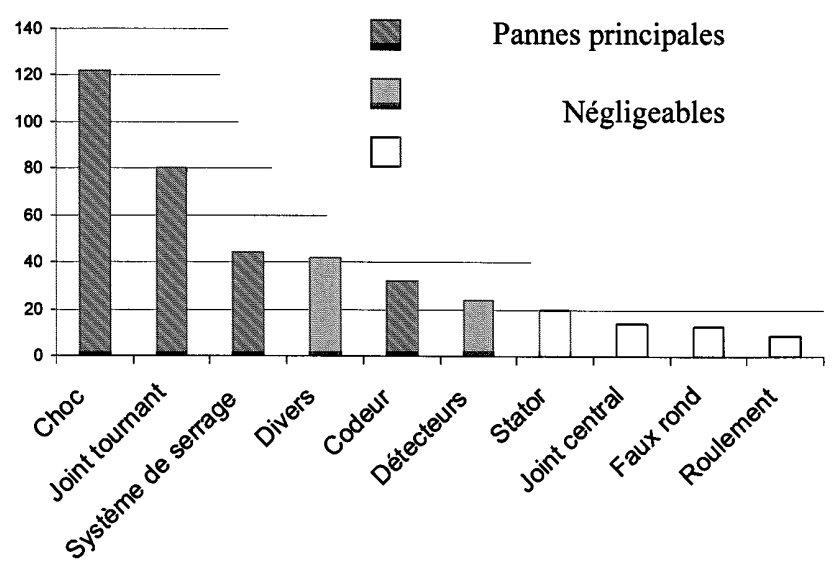

Fig. 5. Histogramme des pannes (données COMAU + PCI).

milieu industriel, les mêmes matériaux, et ne pratiquent pas les mêmes opérations. Il s'avère que ces aspects sont importants par l'influence des paramètres comme le débit de copeaux, le nombre de changements d'outils, l'intensité des régimes, la qualité des bruts et des systèmes de positionnement, le respect des règles de fonctionnement caractéristiques pour chaque entreprise.

La figure 5 identifie les pannes les plus souvent rencontrées : les pannes générées par le joint tournant, celles du système de serrage des porte-outils et celles du codeur. En première place se trouvent pourtant les pannes pour lesquelles l'événement initiateur a été une collision avec le magasin d'outils ou un choc subi par la broche.

Il y a évidemment un enchevêtrement entre les différents sous-systèmes et une logique assez compliquée qui définit et relie l'élément ou l'événement initiateur de la panne au sous-système défaillant. Il fallait suivre donc une démarche rigoureuse pour pouvoir identifier les pannes, les symptômes et les mesures susceptibles de constituer les paramètres d'un système de suivi de la santé des électrobroches.

La figure 6 présente une optique capable de classer les différentes notions et de tracer une cartographie des événements pouvant conduire à la panne d'une électrobroche [6].

\section{Définition d'un système de suivi périodique}

La figure 7 présente les relations causes-effets trouvées par la logique d'analyse présentée. Les mesures pouvant nous fournir des informations pertinentes sont :

- accélérations et déplacements capables de caractériser les vibrations de l'ensemble tournant soit par mesure directe (capteurs de déplacement) soit par mesure indirecte (accéléromètres fixés sur éléments fixes, paliers, carter). Dans le deuxième cas il se pose le problème de l'emplacement optimum des capteurs ;

- température des paliers susceptible de signaler une panne du circuit de refroidissement et/ou lubrification, une usure prononcée des roulements ou un

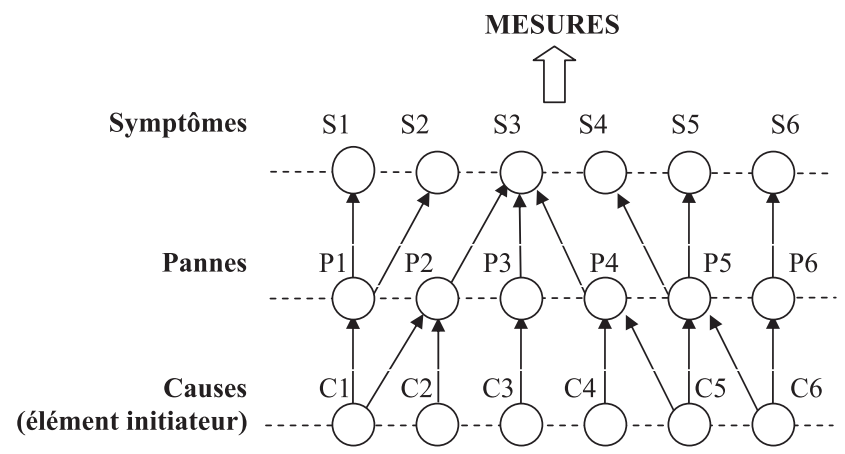

Fig. 6. Logique de l'analyse des pannes [6].

délignage important, etc. Ce capteur est installé sur un certain nombre de modèles des broches fournies par FISCHER;

- intensité du courant moteur, température du stator, et vitesse mesurée, signaux disponibles aussi via le variateur de l'électrobroche;

- les signaux du codeur pourront aussi être utiles; une étude préliminaire a montré que la précision des deux capteurs intégrés est suffisante pour détecter des problèmes de faux rond ou vibrations du palier arrière via un traitement de signal adéquat.

Les mesures de déplacement et accélération seront orientées vers la détermination des indicateurs de comportement de l'axe de la broche : faux rond statique, faux rond dynamique, indicateurs de surveillance vibratoire. La mesure de température sera faite dans des conditions identiques; il faut mesurer l'échauffement de la broche dans les mêmes conditions de sollicitation. Le codeur peut donner des informations sur les vibrations du palier arrière et sur son bon fonctionnement à basse et haute vitesse.

Une procédure de mesure rapide de la rigidité radiale statique de la broche a été également mise en place, afin de suivre l'évolution de ce paramètre pendant le cycle de vie de la broche. Le système de serrage pourra être testé avec un système de mesure de la force de serrage et par une estimation de la précision.

Le système de mesure proposé (Fig. 8) comporte une partie opérative dotée de capteurs qui pourra se monter en quelques minutes sur le nez de la broche. En même temps, une boîte de dérivation et traitement de signal installée dans l'armoire de puissance pourra fournir les signaux évoqués. Les éléments du système de mesure et la mise au point des procédures se trouvent en phase de validation. Cette étape préliminaire est réalisée dans le LSIS utilisant une broche WEISS ayant le même variateur que les broches FISCHER et une broche prototype fabriquée dans le laboratoire.

Ce système de mesure périodique et les procédures associées seront mis au profit bientôt par une campagne d'essais sur le site de COMAU Systèmes France. 


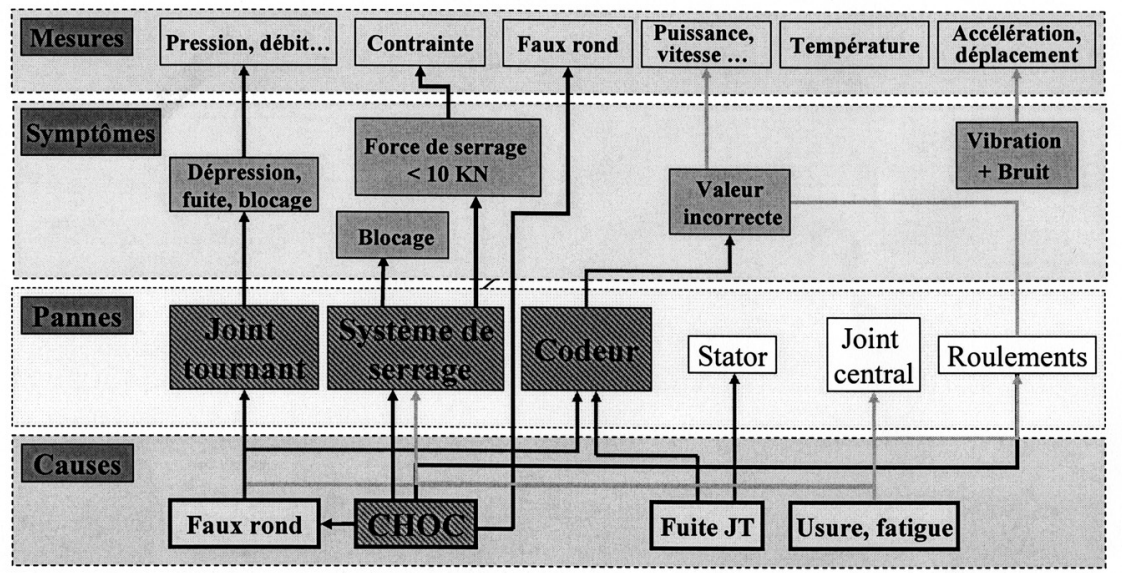

Fig. 7. Relation de causes à effets dans le fonctionnement des électrobroches.

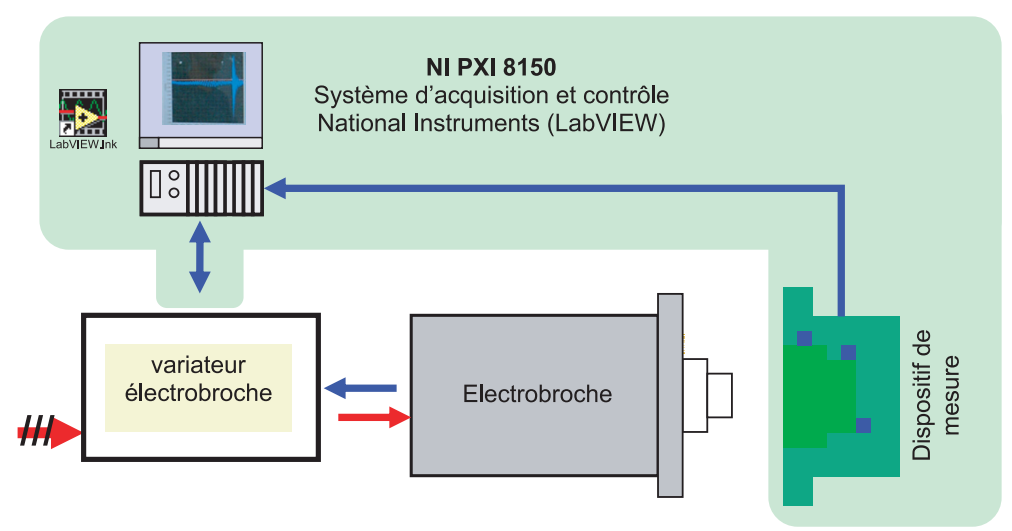

Fig. 8. Définition d'un système de mesure portable.

Cette opération vise la collecte de données et le tracé des historiques de broches afin de mettre au point une procédure finale de suivi périodique, capable d'améliorer la démarche de maintenance actuellement pratiquée.

\section{Références}

[1] R. Walter, Monitoring of High Speed Milling Spindles - Requirements, Concepts and Solutions, Weiss Spindeltechnologie GmbH, Schweinfurt, Germany
[2] M. Kaufeld, S. Torbaty, Rationalisation de l'usinage très grande vitesse, SOFETEC, ISBN-1281-7260, 1994

[3] M. Chang Kang, J. Suk Kim, J. Ha Kim, A monitoring technique using a multisensor in high speed machining, J. Mat. Proc. Tech. 113 (2001) 331-336

[4] S. Mas, Les réseaux de neurones pour le diagnostic : application à l'analyse prédictive de défaillance des broches UGV, LSIS, juillet 2003

[5] C. Andruszko, Développement d'un banc d'essais, Mémoire CNAM, Aix-en-Provence, janvier 2004

[6] P. Arques, Diagnostic prédictif de l'état des machines, Masson, 1996 